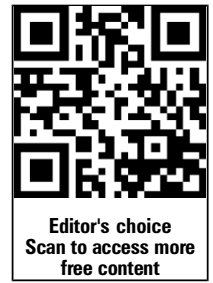

ree content
Handling editor Tore $K$ Kvien

${ }^{1}$ Division of Immunology and Rheumatology, Stanford University, Palo Alto

California, USA

${ }^{2}$ Department of Rheumatology, University of Texas,

Southwestern Medical Center,

Dallas, Texas, USA

${ }^{3}$ Desert Medical Advances,

Palm Desert, California, USA

${ }^{4}$ Eli Lilly and Company,

Indianapolis, Indiana, USA

\section{Correspondence to}

Dr Mark Genovese,

Division of Immunology and

Rheumatology, Stanford

University, 1000 Welch Rd

\#203, Palo Alto, CA 94304,

USA;

Genovese@Stanford.edu

Received 3 October 2012 Revised 21 November 2012 Accepted 27 November 2012

Published Online First

25 December 2012

\title{
Tabalumab, an anti-BAFF monoclonal antibody, in patients with active rheumatoid arthritis with an inadequate response to TNF inhibitors
}

\author{
Mark C Genovese, ${ }^{1}$ Roy M Fleischmann, ${ }^{2}$ Maria Greenwald, ${ }^{3}$ Julie Satterwhite, ${ }^{4}$ \\ Melissa Veenhuizen, ${ }^{4}$ Li Xie, ${ }^{4}$ Pierre-Yves Berclaz, ${ }^{4}$ Stephen Myers, ${ }^{4}$ Olivier Benichou ${ }^{4}$
}

\section{ABSTRACT}

Objective To evaluate the efficacy and safety of tabalumab, a monoclonal antibody that neutralises membrane-bound and soluble B-cell activating factor (BAFF), in patients with active rheumatoid arthritis (RA) who showed inadequate response to tumour necrosis factor (TNF) inhibitors.

Methods Patients on stable methotrexate and with inadequate response to one or more TNF inhibitors were randomised to placebo $(n=35), 30 \mathrm{mg}$ tabalumab $(n=35)$ or $80 \mathrm{mg}$ tabalumab $(n=30)$ given intravenously at 0, 3 and 6 weeks. The primary outcome was the proportion of patients achieving an American College of Rheumatology 50\% response (ACR50) at week 16 (all tabalumab-treated patients vs placebo).

Results At week 16, no significant differences were observed in the combined tabalumab group versus placebo in ACR50 (12.7\% vs 2.9\%, $p=0.101)$ or ACR20 response rates ( $27.0 \%$ vs $17.1 \%, p=0.198)$. However, significant differences between the combined tabalumab group and placebo were observed at earlier time points for ACR20, ACR50 and Disease Activity Score in 28 joints (DAS28)-C-reactive protein (CRP) reduction. Treatment-emergent adverse events (AEs) were similar with $30 \mathrm{mg}$ tabalumab (65.7\%), $80 \mathrm{mg}$ tabalumab $(76.7 \%)$ and placebo $(71.4 \%)$, although certain events occurred more often with tabalumab than placebo (eg, infection, anaemia and gastrointestinal events). Serious AEs occurred in two $(6.7 \%)$ patients receiving $80 \mathrm{mg}$ tabalumab and three $(8.6 \%)$ receiving placebo, with one serious infection in the placebo group. Initial increases in total and mature B cells were followed by progressive decreases, despite declines in serum tabalumab.

Conclusions At week 16, the primary end point was not achieved, but an indication of efficacy was observed at earlier time points. Safety findings for tabalumab were consistent with other biological RA therapies.

Clinical trial registration number NCT00689728.

\section{INTRODUCTION}

Rheumatoid arthritis (RA) is a serious autoimmune disease, affecting $\sim 1 \%$ of the population. ${ }^{1}$ Biological therapies that selectively target key molecules associated with joint inflammation, such as tumour necrosis factor (TNF) inhibitors, are effective options for RA treatment. ${ }^{2}$ Many patients continue to have active disease despite treatment with biological disease-modifying antirheumatic drugs
(bDMARDs), including TNF inhibitors, and are in need of new treatment options. ${ }^{3-6}$

B-cell activating factor (BAFF) is a ligand in the TNF family that exists in a membrane-bound and soluble form and is required for B-cell survival as they leave the bone marrow and enter the periphery. $^{7}{ }^{8}$ BAFF prevents apoptosis of B cells, regulates $\mathrm{B}$-cell homoeostasis, and maintains peripheral tolerance. ${ }^{9}$ It also acts as a co-stimulatory cytokine (with a proliferation-inducing ligand (APRIL)) to promote class switching. ${ }^{10}$ In patients with RA, increased BAFF expression in the sera and synovial fluid may increase the likelihood of B-cell survival; thus, neutralising BAFF is an alternative therapeutic approach to targeting B cells. ${ }^{11} 12$

Tabalumab is a human anti-BAFF monoclonal antibody that neutralises both biologically active forms (membrane-bound and soluble) of BAFF. ${ }^{13}$ In an initial dose-ranging study, tabalumab reduced RA signs and symptoms in subjects naïve to bDMARDs, such as TNF inhibitors. ${ }^{14}$

The present study compared the efficacy and safety of tabalumab with placebo in patients with active RA who had an inadequate response or intolerance to TNF inhibitors (TNF-IR).

\section{PATIENTS AND METHODS}

\section{Patients}

Patients with active RA were recruited from 45 centres in Argentina, Austria, Belgium, Brazil, Canada, Germany, Mexico, Poland, Puerto Rico and the USA. All patients provided voluntary written informed consent. The study was approved by local institutional review boards in accordance with the Declaration of Helsinki, good clinical practice, and applicable laws and regulations.

Adult (18-75 years) men and non-pregnant women with an RA diagnosis defined by the American Rheumatism Association 1987 revised criteria $^{15}$ and who had a history of inadequate response or intolerance to one or more TNF inhibitors were enrolled. Major inclusion criteria were active disease defined by $\geq 5 / 28$ swollen and $\geq 5 / 28$ tender joints, with a C-reactive protein (CRP) $\geq$ the upper limit of normal (ULN, $1.0 \mathrm{mg} / \mathrm{dl}$ ) or an erythrocyte sedimentation rate $(E S R) \geq 28 \mathrm{~mm} / \mathrm{h}$. In addition, patients had been taking methotrexate (MTX) for $\geq 12$ weeks (stable dose of $7.5-25 \mathrm{mg}$ / week for $\geq 8$ weeks before baseline), and had a 
positive rheumatoid factor (RF) and/or an anti-cyclic citrullinated peptide (anti-CCP) test.

Major exclusion criteria were: previous use of B-cell-targeted therapy; taking a live vaccine within 3 months of enrolment; serious bacterial infection within 6 months of enrolment; herpes zoster within 3 months of enrolment; evidence of HIV, or hepatitis $\mathrm{B}$ or $\mathrm{C}$ infection; active tuberculosis; serum IgG, IgM, or IgA concentration below the lower limit of normal (LLN); history of malignancy within 5 years; any unstable comorbidity; a recent or ongoing infection that posed an unacceptable risk according to the investigator; oral corticosteroid use of $>10 \mathrm{mg} /$ day prednisone or its equivalent within 4 weeks of enrolment; parenteral corticosteroid use within 4 weeks of baseline; use of oral DMARDs other than MTX, hydroxychloroquine or sulfasalazine at a stable dose for $\geq 8$ week before baseline.

\section{Study design}

This was a 16-week, randomised, double-blind, placebocontrolled, phase 2, parallel-group study. Eligible patients were maintained on background MTX at their stable, pre-study dose and were randomly assigned by an interactive voice-response system to receive $30 \mathrm{~min}$ infusions of tabalumab $30 \mathrm{mg}$, tabalumab $80 \mathrm{mg}$, or placebo intravenously at weeks 0,3 and 6. Doses were selected from an efficacious dose range previously demonstrated in TNF inhibitor-naïve patients. ${ }^{14}$

Efficacy and safety were evaluated at baseline, weeks 1, 3, 6, 9, 12 and 16. As an amendment to the study, non-responders (patients with $<20 \%$ improvement in either their tender or swollen joint counts ( 28 count) by week 16 ) were given the opportunity to receive an open-label, $30 \mathrm{~min}$ infusion of $80 \mathrm{mg}$ tabalumab as rescue therapy at week 16. This amendment changed the time of the primary end point from 24 to 16 weeks, as it was felt that the addition of rescue therapy would bias the analysis at 24 weeks.

\section{End points}

The primary efficacy end point was the proportion of patients who achieved an American College of Rheumatology 50\% (ACR50) response at week $16 .{ }^{16}$ Key secondary efficacy measures included the proportion of patients achieving ACR20, ACR50 and ACR70, the mean percentage improvement in Disease Activity Score in 28 joints (DAS28)-C-reactive protein (CRP), and the percentage of patients achieving DAS28-CRP $<3.2$ and $<2.6$ at each visit, as well as the percentage of moderate or good responders based on the European League Against Rheumatism (EULAR) responder criteria. ${ }^{17}$ Additional study objectives were to evaluate pharmacodynamics of disease-related biomarkers after study drug administration, to explore potential associations between selected biomarkers and disease activity measures, and to further characterise tabalumab pharmacokinetics.

Blood samples for determination of serum tabalumab concentrations and pharmacodynamic measures (ie, B-cell counts, CRP, ESR and immunoglobulins) were collected at baseline and subsequent clinic visits and were assayed using validated methods.

Adverse events (AEs) and serious adverse events (SAEs) were recorded at each visit. To assess immunogenicity, blood samples were collected at the three infusion visits (weeks 0,3 and 6) and, for late onset of immunogenicity, at weeks 24,32 and 40. Vital signs and laboratory tests, including chemistry, haematology and urinalysis panels, were collected at each visit.

\section{Statistical methods}

Sample sizes were determined assuming a $23 \%$ absolute difference based on previous trial data ${ }^{14}$ to provide $83 \%$ statistical power to detect a significant difference in ACR50 responses between placebo and the combined tabalumab group (without continuity correction, one-sided hypothesis, 5\% type I error rate). Assuming a 10\% response rate in the placebo group and $33 \%$ in the treatment groups at week 16 , it was calculated that approximately 33 patients were needed per arm (33, placebo; 66, combined tabalumab).

Efficacy analyses were performed using a modified intentto-treat population (mITT) (all randomised patients who received $\geq 1$ study-drug dose (tabalumab or placebo) and who have $\geq 1$ post-baseline efficacy assessment). At the end of the study, data integrity issues were discovered at one study site with two patients in the $80 \mathrm{mg}$ group; efficacy analyses were based on mITT population, excluding patients from this site. Safety analyses included all patients who received any study drug.

The primary efficacy analysis compared the percentage of patients achieving an ACR50 response in the combined 30 and $80 \mathrm{mg}$ tabalumab groups versus placebo at week 16. A one-sided Fisher exact test was used for this analysis, as well as ACR20 and ACR70 analyses at each time point $(\alpha=0.05)$. For DAS28-CRP changes from baseline, one-sided comparisons of tabalumab (the two dose groups combined) versus placebo were performed using an analysis of covariance (ANCOVA) test, with treatment and baseline as fixed factors $(\alpha=0.05)$. The percentage

Table 1 Patient disposition, demographics and baseline characteristics

\begin{tabular}{|c|c|c|c|}
\hline & \multirow[b]{2}{*}{$\begin{array}{l}\text { Placebo } \\
\text { (N=35) }\end{array}$} & \multicolumn{2}{|l|}{ Tabalumab } \\
\hline & & $\begin{array}{l}30 \mathrm{mg} \\
(\mathrm{N}=35)\end{array}$ & $\begin{array}{l}80 \mathrm{mg} \\
(\mathrm{N}=30)\end{array}$ \\
\hline \multicolumn{4}{|l|}{ Patient disposition } \\
\hline Completed the study & $29(82.9)$ & $30(85.7)$ & $24(80.0)$ \\
\hline Discontinued study medication & $6(17.1)$ & $5(14.3)$ & $6(20.0)$ \\
\hline \multicolumn{4}{|l|}{ Reason for discontinuation } \\
\hline Adverse event & 0 & $1(2.9)$ & $1(3.3)$ \\
\hline Lost to follow-up & 0 & 0 & 0 \\
\hline Consent withdrawal & $3(8.6)$ & $4(11.4)$ & $4(13.3)$ \\
\hline Protocol violation & $1(2.9)$ & 0 & 0 \\
\hline Lack of efficacy & $2(5.7)$ & 0 & $1(3.3)$ \\
\hline Received rescue therapy & $20(57.1)$ & $12(34.3)$ & $11(36.7)$ \\
\hline \multicolumn{4}{|c|}{ Demographics and baseline characteristics } \\
\hline Age (years) & $52.2 \pm 11.5$ & $52.4 \pm 13.0$ & $52.7 \pm 14.1$ \\
\hline Women & 32 (91.4) & $28(80.0)$ & $26(86.7)$ \\
\hline Caucasian & $21(60.0)$ & $26(74.3)$ & $20(66.7)$ \\
\hline BMI $\left(\mathrm{kg} / \mathrm{m}^{2}\right)$ & $27.8 \pm 5.0$ & $27.4 \pm 6.0$ & $30.0 \pm 8.5$ \\
\hline Weight (kg) & $72.8 \pm 13.9$ & $75.1 \pm 18.0$ & $79.2 \pm 27.0$ \\
\hline Disease duration (years) & $10.9 \pm 8.3$ & $10.4 \pm 6.7$ & $9.8 \pm 7.6$ \\
\hline Swollen joint count (28) & $14.5 \pm 5.7$ & $12.6 \pm 5.7$ & $11.5 \pm 6.5^{*}$ \\
\hline Tender joint count (28) & $18.3 \pm 7.1$ & $17.2 \pm 6.8$ & $16.1 \pm 9.0$ \\
\hline HAQ-DI & $1.8 \pm 0.5$ & $1.7 \pm 0.6$ & $1.6 \pm 0.7$ \\
\hline $\mathrm{CRP}(\mathrm{mg} / \mathrm{dl})$ & $2.2 \pm 2.5$ & $1.7 \pm 2.0$ & $2.0 \pm 2.3$ \\
\hline $\mathrm{ESR}(\mathrm{mm} / \mathrm{h})$ & $52.5 \pm 31.6$ & $44.9 \pm 21.9$ & $51.0 \pm 25.0$ \\
\hline DAS28-CRP & $6.2 \pm 0.9$ & $6.0 \pm 0.9$ & $5.8 \pm 1.3$ \\
\hline MTX dose (mg/week) & $17.1 \pm 4.8$ & $15.6 \pm 4.6$ & $16.8 \pm 4.6$ \\
\hline Currently using prednisone & $24(68.6)$ & $24(68.6)$ & $17(56.7)$ \\
\hline Prednisone dose (mg/week) & $48.4 \pm 18.6$ & $53.8 \pm 16.4$ & $53.5 \pm 17.2$ \\
\hline \multicolumn{4}{|c|}{$\begin{array}{l}\text { Values are mean } \pm S D \text { or number }(\%) \text { of patients. } \\
{ }^{*} p<0.05 \text { vs placebo. }\end{array}$} \\
\hline \multicolumn{4}{|c|}{$\begin{array}{l}\text { BMI, body mass index; CRP, C-reactive protein; DAS28, Disease Activity Score } \\
\text { (based on } 28 \text { joints); ESR, erythrocyte sedimentation rate; HAQ-DI, Health } \\
\text { Assessment Questionnaire-Disability Index; MTX, methotrexate; N, total number of } \\
\text { patients randomised. }\end{array}$} \\
\hline
\end{tabular}


of patients who achieved moderate or good EULAR response was analysed using a one-sided Fisher exact test of tabalumab (the two dose groups combined) versus placebo $(\alpha=0.05)$. Pharmacodynamic measures, including CRP, B cells, ESR and serum IgA, IgG and IgM, were analysed using two-sided, ranked ANCOVA, with treatment as the fixed factor and the standardised rank baseline value as a covariate $(\alpha=0.05)$. Non-responder imputation (NRI) and last-observation-carried-forward methods were used to impute missing data in ACR20/50/70 analyses and other analyses, respectively. Adverse events were summarised using percentages. Tabalumab pharmacokinetic parameters were analysed using a population approach implemented with NONMEM (ICON Development Solutions, Ellicott City, Maryland, USA).

\section{RESULTS}

\section{Patient disposition and characteristics}

One hundred patients were randomised to receive treatment: 35 in the placebo group, 35 in the $30 \mathrm{mg}$ tabalumab group, and 30 in the $80 \mathrm{mg}$ tabalumab group. Of the 100 treated patients, 83 completed the study. Patient disposition, with reasons for discontinuation, is shown in table 1.

Baseline demographics and disease characteristics are also shown in table 1. Patients in the combined tabalumab group had significantly lower swollen joint counts (28 count) than the placebo group (combined tabalumab, 12.1; placebo, 14.5; $\mathrm{p}<0.05)$. All other baseline characteristics were comparable across groups.

\section{Clinical response}

The ACR50 response rate (NRI) in the combined tabalumab group at week 16 was not significantly different from the placebo group $(12.7 \%$ vs $2.9 \%, p=0.101)$ (figure $1 \mathrm{~A}$, table 2 ), nor was the ACR20 response rate $(27.0 \%$ vs $17.1 \%, p=0.198)$ (figure 1B, table 2). ACR20, ACR50 and ACR70 response rates over time are displayed in table 2 and figure 1A-C. ACR2 0 and ACR50 response rates (NRI) were significantly higher in the combined tabalumab group versus placebo at week 6 (ACR20, $38.1 \%$ vs $17.1 \%, p=0.025$; ACR $50,11.1 \%$ vs $0.0 \%, p=0.040)$ and week 9 (ACR20, 41.3\% vs 17.1\%, $p=0.012$; ACR50, 22.2\% vs $0.0 \%, p=0.001)$, but the differences were no longer significant by week 12 (ACR20, 42.9\% vs 25.7\%, p=0.070; ACR50, $14.3 \%$ vs $2.9 \%, p=0.068$ ) (table 2 (week 6 data not shown in table), figure $1 \mathrm{~A}, \mathrm{~B})$. ACR70 response rates were not significantly different at any time point (table 2 , figure $1 \mathrm{C}$ ).

The reduction in DAS28-CRP from baseline was significantly greater for the combined tabalumab group than for the placebo group at weeks 6, 9, 12 and 16 (table 2 (week 6 data not shown in table), figure 1D). A higher percentage of patients in the combined tabalumab group versus placebo achieved
A
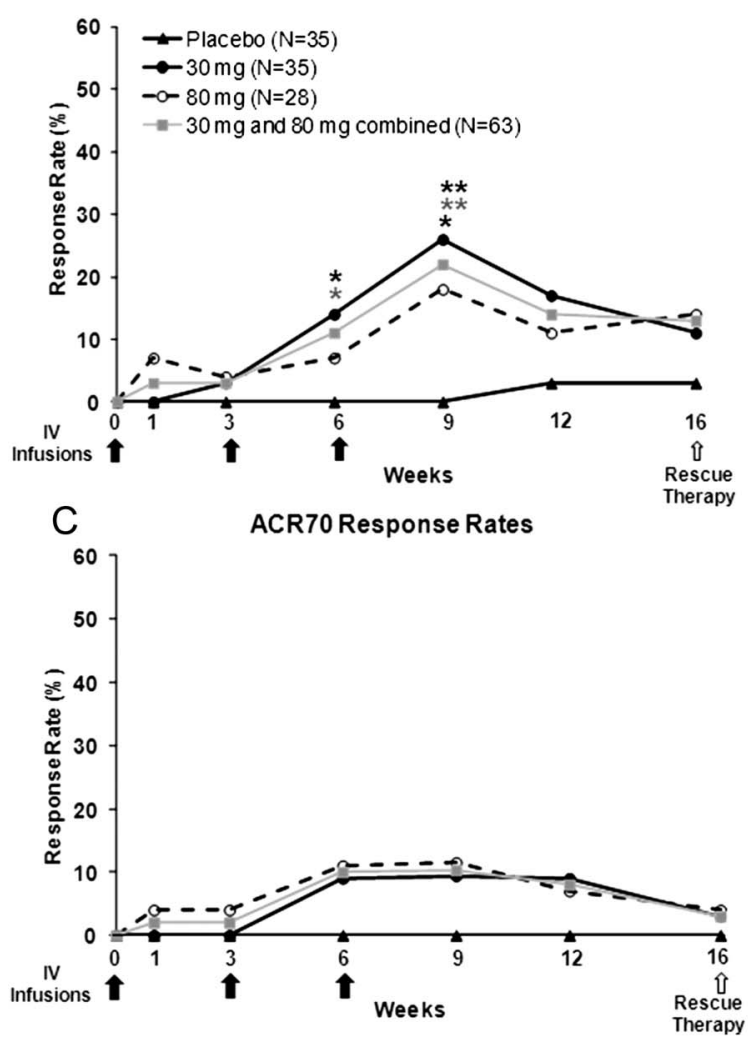

B
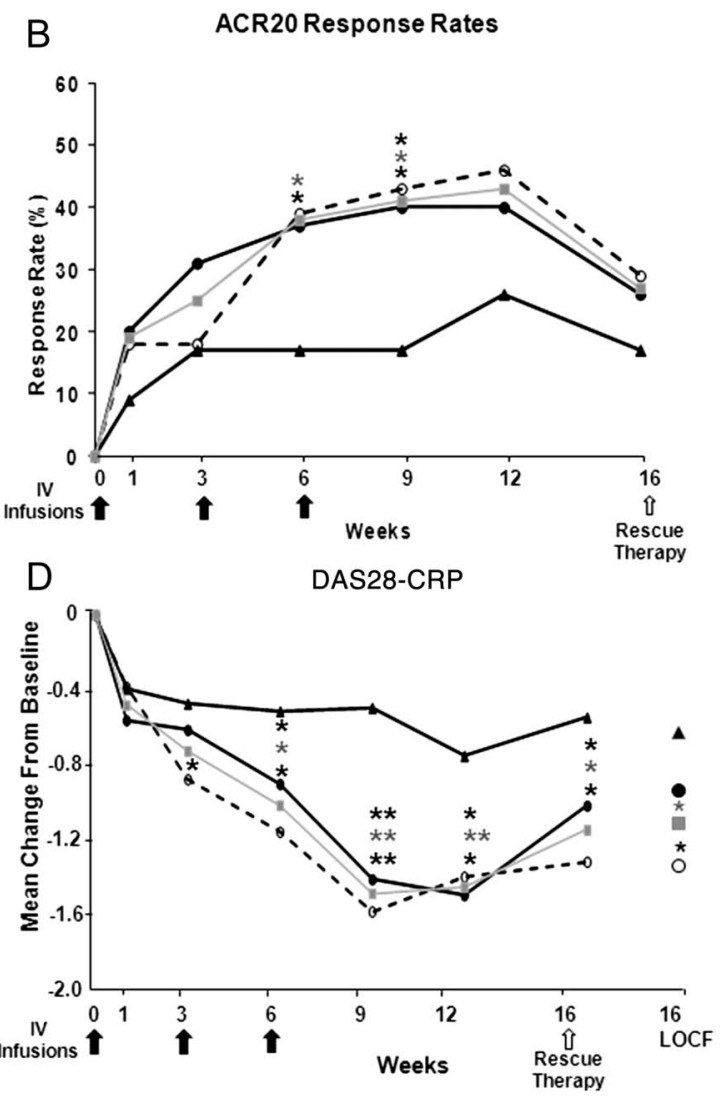

Figure 1 Efficacy measures over 16 weeks. For $(A-C), p$ values are based on a one-sided Fisher exact test that the tabalumab group has more responders than placebo. For (D), $p$ values are based on a one-sided analysis of covariance test of tabalumab (the two dose groups combined) versus placebo with treatment and baseline value in the model. ${ }^{*} p \leq 0.05,{ }^{* *} p \leq 0.001$ vs placebo. (A) ACR50 response rates at each treatment visit with tabalumab versus placebo. (B) ACR20 response rates at each treatment visit with tabalumab vs placebo. (C) ACR70 response rates at each treatment visit with tabalumab versus placebo. (D) Mean change in DAS28-CRP at each treatment visit with tabalumab versus placebo. ACR20, American College of Rheumatology 20 responder index; ACR50, American College of Rheumatology 50 responder index; ACR70, American College of Rheumatology 70 responder index; CRP, C-reactive protein; DAS28, Disease Activity Score based on 28-joint count; IV, intravenous; LOCF, last-observation-carried-forward; NRI, non-responder imputations. 


\begin{tabular}{|c|c|c|c|c|c|c|c|c|c|c|c|c|}
\hline \multirow[b]{3}{*}{ End point } & \multicolumn{4}{|l|}{ Week 9} & \multicolumn{4}{|l|}{ Week 12} & \multicolumn{4}{|l|}{ Week 16} \\
\hline & \multirow[b]{2}{*}{ Placebo $(\mathrm{N}=35$ ) } & \multicolumn{2}{|l|}{ Tabalumab } & \multirow[b]{2}{*}{ p Value } & \multirow[b]{2}{*}{ Placebo $(\mathrm{N}=35)$} & \multicolumn{2}{|l|}{ Tabalumab } & \multirow[b]{2}{*}{ p Value } & \multirow[b]{2}{*}{ Placebo $(\mathrm{N}=35)$} & \multicolumn{2}{|l|}{ Tabalumab } & \multirow[b]{2}{*}{ p Value } \\
\hline & & $30 \mathrm{mg}(\mathrm{N}=35)$ & $80 \mathrm{mg}(\mathrm{N}=28)$ & & & $30 \mathrm{mg}(\mathrm{N}=35)$ & $80 \mathrm{mg}(\mathrm{N}=28)$ & & & $30 \mathrm{mg}(\mathrm{N}=35)$ & $80 \mathrm{mg}(\mathrm{N}=28)$ & \\
\hline \multicolumn{13}{|l|}{ ACR20*† } \\
\hline $\mathrm{n} / \mathrm{N}(\%)$ & $6 / 35(17.1)$ & $14 / 35(40.0)$ & $12 / 28(42.9)$ & 0.012 & $9 / 35(25.7)$ & $14 / 35(40.0)$ & $13 / 28(46.4)$ & 0.070 & $6 / 35(17.1)$ & $9 / 35(25.7)$ & $8 / 28(28.6)$ & 0.198 \\
\hline \multicolumn{13}{|l|}{ ACR50* $†$} \\
\hline $\mathrm{n} / \mathrm{N}(\%)$ & $0 / 35(0.0)$ & $9 / 35(25.7)$ & $5 / 28(17.9)$ & 0.001 & $1 / 35(2.9)$ & $6 / 35(17.1)$ & $3 / 28(10.7)$ & 0.068 & $1 / 35(2.9)$ & $4 / 35$ (11.4) & $4 / 28(14.3)$ & 0.101 \\
\hline \multicolumn{13}{|l|}{ ACR70* } \\
\hline $\mathrm{n} / \mathrm{N}(\%)$ & $0 / 35(0.0)$ & $3 / 35(8.6)$ & $3 / 28(10.7)$ & 0.065 & $0 / 35(0.0)$ & $3 / 35(8.6)$ & $2 / 28(7.1)$ & 0.104 & $0 / 35(0.0)$ & $1 / 35(2.9)$ & $1 / 28(3.6)$ & 0.411 \\
\hline \multicolumn{13}{|l|}{ DAS28-CRP $<3.2^{*}$} \\
\hline $\mathrm{n} / \mathrm{N}(\%)$ & $1 / 35(3.2)$ & $6 / 35$ (18.8) & $7 / 28(26.9)$ & 0.014 & $1 / 35(3.3)$ & $5 / 35(16.7)$ & $4 / 28(16.7)$ & 0.066 & $0 / 35(0.0)$ & $5 / 35(14.3)$ & 6/28 (21.4) & $0.005 \ddagger$ \\
\hline \multicolumn{13}{|l|}{ DAS28-CRP $<2.6^{*}$} \\
\hline $\mathrm{n} / \mathrm{N}(\%)$ & $1 / 35(3.2)$ & $3 / 35(9.4)$ & $5 / 28(19.2)$ & 0.110 & $0 / 35(0.0)$ & $3 / 35(10.0)$ & $3 / 28$ (12.5) & 0.064 & $0 / 35(0.0)$ & $3 / 35(8.6)$ & $2 / 28(7.1)$ & $0.104 \ddagger$ \\
\hline \multicolumn{13}{|l|}{ DAS28-CRP§ } \\
\hline $\mathrm{N}$ & 31 & 32 & 25 & & 30 & 30 & 23 & & 34 & 35 & 26 & \\
\hline Mean change & $-0.50 \pm$ & $-1.42 \pm$ & $-1.59 \pm$ & & $-0.76 \pm$ & $-1.50 \pm$ & $-1.40 \pm$ & & $-0.61 \pm$ & $-0.91 \pm$ & $-1.29 \pm$ & \\
\hline SD & 1.06 & 1.22 & 1.25 & $<0.001$ & 0.91 & 1.10 & 1.16 & $<0.001$ & 1.04 & 1.14 & 0.93 & $0.016 \ddagger$ \\
\hline \multicolumn{13}{|l|}{ CRPף } \\
\hline $\mathrm{N}$ & 31 & 32 & 26 & 0.038 & 30 & 30 & 24 & & 35 & 35 & 27 & \\
\hline Median $\%$ change & -8.07 & -32.98 & -23.69 & & -29.78 & -15.97 & -22.55 & 0.996 & -29.15 & -5.72 & -20.19 & $0.650 \ddagger$ \\
\hline \multicolumn{13}{|c|}{ EULAR response $(\text { good }+ \text { moderate })^{*}$} \\
\hline $\mathrm{n} / \mathrm{N}(\%)$ & $9 / 31(29.0)$ & $22 / 32(68.8)$ & $17 / 25(68.0)$ & $<0.001$ & $15 / 30(50.0)$ & $21 / 30(70.0)$ & $15 / 23(65.2)$ & 0.085 & $15 / 34(44.1)$ & $13 / 35(37.1)$ & $17 / 26(65.4)$ & $0.398 \ddagger$ \\
\hline Regional subanalysis & Week 9 (Observec & & & & Week 12 (Observ & & & & Week 16 (NRI) & & & \\
\hline \multicolumn{13}{|l|}{ ACR20 (US) } \\
\hline $\mathrm{n} / \mathrm{N}(\%)$ & $3 / 12(25.0)$ & $6 / 16(37.5)$ & $5 / 14(35.7)$ & NC & $6 / 12(50.0)$ & $4 / 16(25.0)$ & $3 / 12(25.0)$ & NC & $3 / 14$ (21.4) & $5 / 18(27.8)$ & 2/16 (12.5) & NC \\
\hline \multicolumn{13}{|l|}{ ACR20 (OUS) } \\
\hline $\mathrm{n} / \mathrm{N}(\%)$ & $3 / 19(15.8)$ & $8 / 16(50.0)$ & $7 / 12(58.3)$ & NC & $3 / 19(15.8)$ & $10 / 15(66.7)$ & 10/12 (83.3) & NC & $3 / 21(14.3)$ & $4 / 17(23.5)$ & $6 / 12(50.0)$ & NC \\
\hline \multicolumn{13}{|l|}{ ACR50 (US) } \\
\hline $\mathrm{n} / \mathrm{N}(\%)$ & $0 / 12(0.0)$ & $4 / 16(25.0)$ & $2 / 14(14.3)$ & NC & 1/12 (8.3) & $2 / 16(12.5)$ & 1/12 (8.3) & NC & $1 / 14(7.1)$ & $1 / 18(5.6)$ & $1 / 16(6.3)$ & $\mathrm{NC}$ \\
\hline \multicolumn{13}{|l|}{ ACR50 (OUS) } \\
\hline $\mathrm{n} / \mathrm{N}(\%)$ & $0 / 19(0.0)$ & $5 / 16(31.3)$ & $3 / 12(25.0)$ & NC & $0 / 19(0.0)$ & $4 / 15(26.7)$ & $2 / 12(16.7)$ & NC & $0 / 21(0.0)$ & $3 / 17$ (17.6) & $3 / 12(25.0)$ & NC \\
\hline
\end{tabular}

\footnotetext{
OUS countries were Argentina $(n=12)$, Austria $(n=7)$, Belgium $(n=1)$, Brazil $(n=15)$, Canada $(n=4)$, Germany $(n=4)$, Poland $(n=6)$ and Puerto Rico $(n=1)$.

US patients $(\mathrm{n}=48)$.

Values are $n / N(\%)$ unless otherwise noted. For the NRI, patients who discontinued from the study prior to week 16 were imputed as non-responders.
Vatient $(\mathrm{m})$.

*One-sided Fisher exact test of tabalumab (the two dose groups combined) versus placebo.

tImputed by NRI.

†Imputed by LOCF (last-observation-carried-forward).

§One-sided ANCOVA test of tabalumab (the two dose groups combined) versus placebo with treatment and baseline value in the model.

ITwo-sided ranked ANCOVA with treatment and the standardised rank baseline value in the model based on mean percentage change in tabalumab (the two dose groups combined) versus placebo.

**For regional subanalysis, data at weeks 9 and 12 are observed, whereas data at the 16-week primary end point are imputed by NRI.

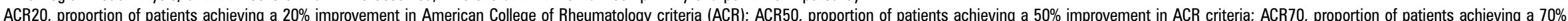

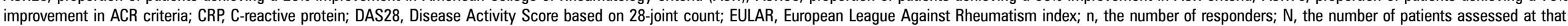
time point; NC, not calculated; NRI, non-responder imputation; OUS, outside of the USA; US, USA.
} 
DAS28-CRP $<3.2$ and $<2.6$; these differences were only significant for DAS28-CRP $<3.2$ at weeks 9 and 16 (table 2). There was a trend for patients in the combined tabalumab group to achieve a good to moderate EULAR during the 16-week, blinded treatment; this difference was only statistically significant at week $6(50.8 \%$ vs $29.4 \%, p=0.036)$ and week $9(68.4 \%$ vs $29.0 \%$, p < 0.001$)$.

In a post hoc analysis, numerically more tabalumab-treated patients outside of the USA (OUS) than in the USA had an ACR20 response (table 2). However, owing to the small sample sizes, data from patients OUS were combined, and no statistical analyses were performed.

\section{Pharmacokinetics}

The tabalumab half-life after administration of the 30 and $80 \mathrm{mg}$ doses was 14.9 and 16.5 days, respectively. Tabalumab concentrations had declined by $97 \%$ at the 16 -week end point (10 weeks after the last dose was administered). Tabalumab concentrations, plotted simultaneously with the time course of ACR response rates, are illustrated in figure 2 .

\section{Biological activity}

At week 16, the median percentage change in ESR from baseline for the placebo $(-11.7 \%)$ and combined tabalumab group $(-23.6 \%)$ did not differ significantly $(p=0.660)$, nor did the median percentage change in CRP between placebo and the combined tabalumab group $(-29.2 \%$ vs $-12.6 \%, p=0.650)$. Some patients with elevated CRP at screening no longer had elevated CRP at baseline. In a post hoc analysis of patients with baseline CRP>ULN $(1.0 \mathrm{mg} / \mathrm{dl})$, the mean percentage change from baseline was $46.4 \%$ for the placebo group and $-6.46 \%$ for the combined tabalumab group at week 16 .

Transient increases in total CD20+ B cells were observed in the combined tabalumab group versus the placebo group at week $1(p<0.05)$. At week 3, the CD20+ B-cell counts began to decrease back to baseline and continued to decline until the end of the observation period (figure 3A). Mature naïve cells
(CD19+, CD27-, IgD+) showed a similar pattern; increases were observed with both 30 and $80 \mathrm{mg}$ tabalumab at week 1 , and, starting at week 3 , these cell counts decreased to baseline or below through week 16 (figure $3 \mathrm{~B}$ ). An increase from baseline in memory cell counts (CD19+, CD27+, IgD-) was observed as early as week 1 and continued until week 12 for both 30 and $80 \mathrm{mg}$ tabalumab versus placebo (figure $3 \mathrm{C}$ ).

For IgM and IgA, statistically significant reductions from baseline were observed in the combined tabalumab group versus the placebo group at week $16(p<0.05)$. For $\operatorname{IgG}$, decreases from baseline for the combined tabalumab group were comparable to those for the placebo group at week 16 . Three patients had reductions in serum IgM that fell below LLN: two patients in the $80 \mathrm{mg}$ group (with no AEs reported) and one patient in the $30 \mathrm{mg}$ group with an $\mathrm{AE}$ of sinusitis.

\section{Safety}

Table 3 summarises AEs during the 16-week treatment period. Two patients discontinued because of an AE (ovarian cyst $(80 \mathrm{mg})$; arrhythmia $(30 \mathrm{mg})$ ). Five patients experienced an SAE: three in the placebo group (ST-segment depression, chest injury and gastroenteritis, one patient each) and two in the combined tabalumab group (RA worsening and newly diagnosed Crohn's disease, one patient each). No patients died during the study. Overall, the rate of AEs was similar across the groups; the majority of AEs were mild to moderate in severity. The most commonly reported AEs for the combined tabalumab group versus placebo were RA worsening (10.8\% vs $25.7 \%$ ), upper respiratory tract infection $(7.7 \%$ vs $0 \%)$, headache $(6.2 \%$ vs $5.7 \%$ ) and upper abdominal pain (6.2\% vs $0 \%$ ). After the last dose of study drug in the combined tabalumab and placebo groups, RA worsening was reported within a similar time frame (25.3 and 27.4 days, respectively) and at a similar frequency ( $46 \%$ and $45 \%$, respectively). There were more infections in the $30 \mathrm{mg}(31.4 \%)$ and $80 \mathrm{mg}(30.0 \%)$ groups than in the placebo group (20.0\%). No tuberculosis, herpes zoster, fungal infections or opportunistic infections were observed in

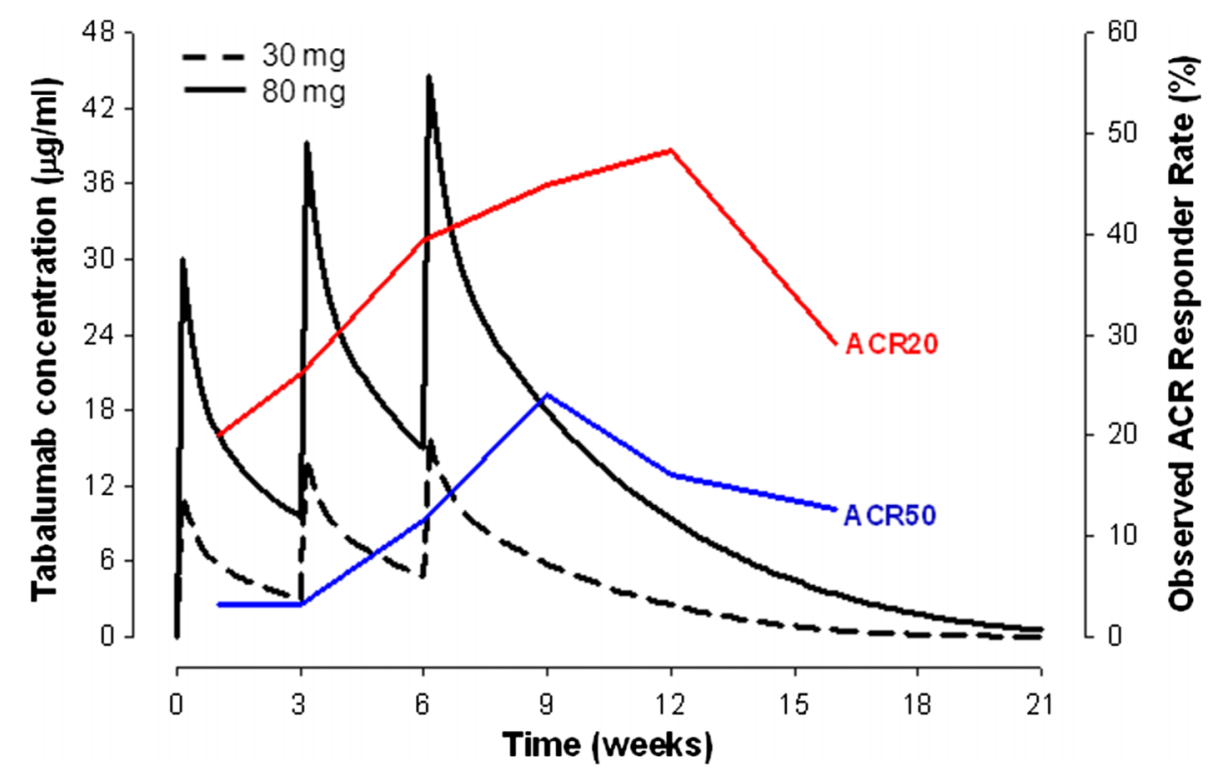

Figure 2 Time course of tabalumab concentrations and ACR20 and ACR50 response rates over time for both 30 and $80 \mathrm{mg}$ doses. The solid black line represents tabalumab concentrations over time for the $80 \mathrm{mg}$ dose. The dashed black line represents tabalumab concentrations over time for the $30 \mathrm{mg}$ dose. The upper grey line (online only: red line) represents ACR20 response rate for the combined 30/80 mg doses. The lower black line (online only: blue line) represents ACR50 response rate for the combined 30/80 mg doses. ACR20, American College of Rheumatology 20 responder index; ACR50, American College of Rheumatology 50 responder index. 

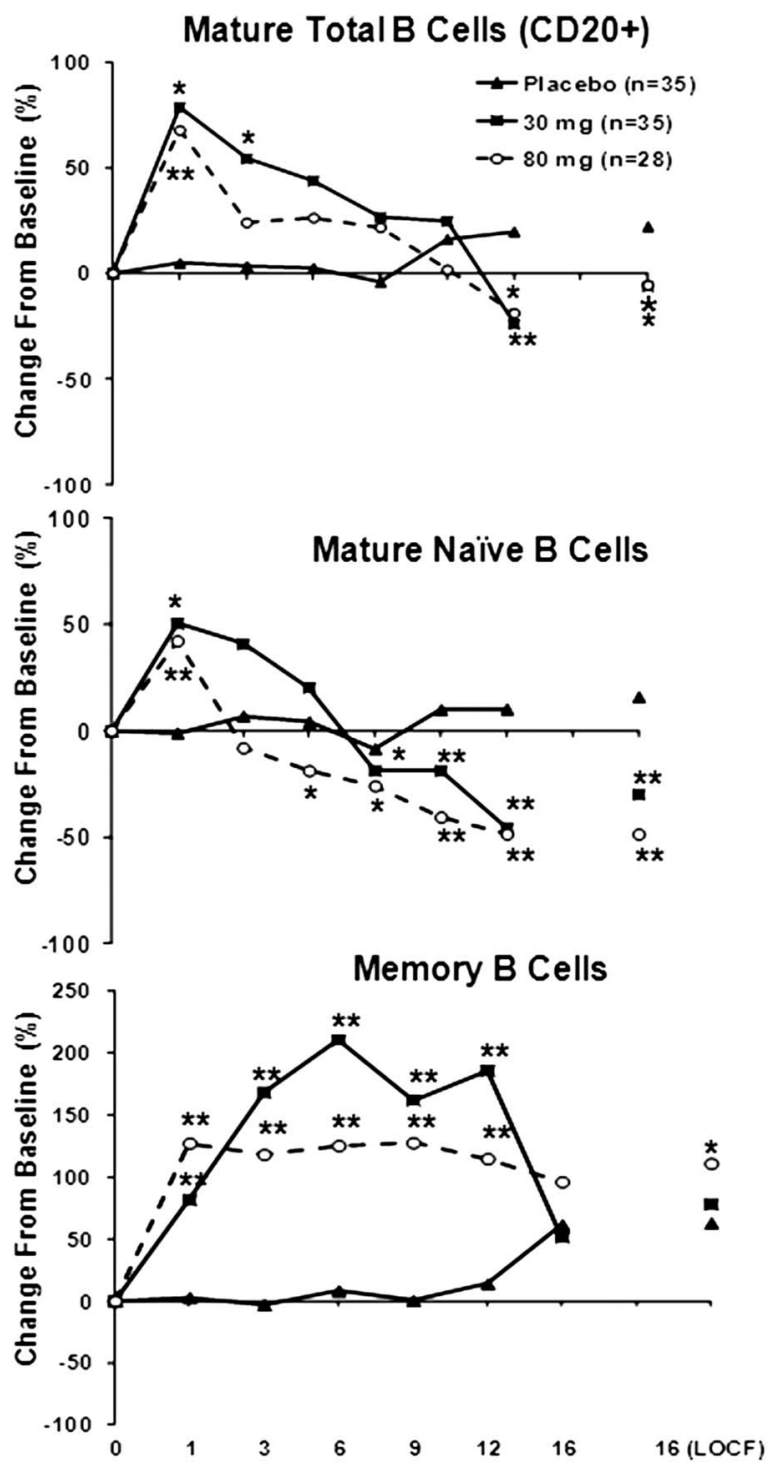

Figure 3 Time course of percentage change in B cells. The $p$ values are based on the one-sided ranked analysis of covariance test of tabalumab versus Placebo, with treatment as the fixed factor and the standardised baseline value as a covariate. ${ }^{*} p \leq 0.05,{ }^{* *} p \leq 0.001$ versus placebo. (A) Change in mature total $B$ cells (CD20) with tabalumab versus placebo. $(B)$ Change in mature naïve $B$ cells with tabalumab versus placebo. (C) Change in memory $B$ cells with tabalumab versus placebo. LOCF, last-observation-carried-forward.

this small number of patients over a short time course. No relationship was observed between changes in serum Ig or B cells and AEs.

No clinically significant differences between treatment groups were observed in laboratory variables, vital sign measurements or ECGs.

\section{Immunogenicity}

No treatment-emergent anti-drug antibodies were detected in patients given tabalumab during the treatment period. One patient (30 mg group) had a transient titre detected (1:20) 42 weeks after the last dose of study drug, and the sample was negative for neutralising antibodies. No anti-drug antibodies were detected in the $80 \mathrm{mg}$ group. Anti-tabalumab antibody status was not associated with AEs.

Table 3 Safety overview: most frequent TEAEs (occurring in $\geq 5 \%$ of patients in any group)

\begin{tabular}{llll}
\hline & & Tabalumab & \\
\cline { 3 - 4 } Adverse event & $\begin{array}{l}\text { Placebo } \\
\text { (N=35) }\end{array}$ & $\begin{array}{l}\mathbf{3 0} \mathbf{~ m g} \\
\text { (N=35) }\end{array}$ & $\begin{array}{l}\mathbf{8 0 ~} \mathbf{~ m g} \\
\text { (N=30) }\end{array}$ \\
\hline Death & 0 & 0 & 0 \\
SAEs & $3(8.6)$ & 0 & $2(6.7)$ \\
Discontinued due to AE & 0 & $1(2.9)$ & $1(3.3)$ \\
TEAEs & $25(71.4)$ & $23(65.7)$ & $23(76.7)$ \\
RA (worsening/flare) & $9(25.7)$ & $4(11.4)$ & $3(10.0)$ \\
Headache & $2(5.7)$ & $1(2.9)$ & $3(10.0)$ \\
Upper respiratory tract infection & 0 & $2(5.7)$ & $3(10.0)$ \\
Anaemia & 0 & 0 & $3(10.0)$ \\
Abdominal pain (upper) & 0 & $2(5.7)$ & $2(6.7)$ \\
Intervertebral disc protrusion & 0 & 0 & $2(6.7)$ \\
Nausea & 0 & 0 & $2(6.7)$ \\
Hypertension & $2(5.7)$ & $2(5.7)$ & $1(3.3)$ \\
Bursitis & 0 & $2(5.7)$ & $1(3.3)$ \\
Pyrexia & 0 & $2(5.7)$ & $1(3.3)$ \\
Nasopharyngitis & $2(5.7)$ & $3(8.6)$ & 0 \\
Hypokalaemia & $2(5.7)$ & $1(2.9)$ & 0 \\
Rhinitis & $2(5.7)$ & $1(2.9)$ & 0 \\
Alopecia & 0 & $2(5.7)$ & 0 \\
Rash papular & 0 & $2(5.7)$ & 0 \\
Sinusitis & 0 & $2(5.7)$ & 0 \\
\hline Vals & & &
\end{tabular}

Values are number (\%) of patients.

$A E$, adverse event; $R A$, rheumatoid arthritis; $S A E$, serious adverse event; TEAE, treatment-emergent adverse event.

\section{DISCUSSION}

This trial examined the efficacy and safety of tabalumab, an anti-BAFF monoclonal antibody, when added to a stable background dose of MTX in TNF-IR patients with active RA. The primary end point was not met, as tabalumab and placebo showed similar ACR50 response rates at week 16.

Although the primary end point was not met, clinical responses were observed early in the study and appeared to peak at week 9 as measured by ACR50 and ACR20 responses and decreases in DAS28-CRP. The reduction in efficacy after week 9 occurred after the last tabalumab infusion and coincided with a notable decline in tabalumab concentrations. This may suggest that the decline in efficacy resulted from the decline in circulating tabalumab concentrations.

A previous phase 2 tabalumab study, which used the same route of administration and dosing frequency, demonstrated that all tabalumab doses (30, 60 and $160 \mathrm{mg}$ ) were significantly more effective than placebo, with improvements maintained up through week $24 .{ }^{14}$ That study enrolled bDMARD-naïve patients, whereas the present study enrolled TNF-IR patients who had already failed one biological treatment and had relatively severe RA. In addition, the two studies differed regionally: the previous tabalumab trial was conducted in one Eastern European country (Romania), and the present study was conducted in 10 countries in North and South America and Europe. The regional data presented in table 2 show that fewer tabalumab-treated patients in the USA achieved ACR20. This may reflect regional differences; however, sample sizes are too small to draw any definitive conclusions. Importantly, the present study offered a one-time rescue dose at week 16 . The addition of rescue altered the timing of the primary analysis from 24 to 16 weeks to avoid the possibility of major bias 8 weeks after a rescue dose. Rescue was not an option in the 
other tabalumab trial. It has been shown that the timing of the rescue dose may influence physicians and patients, and may affect disease activity assessments at the time at which eligibility for rescue is determined. ${ }^{18}$ Ultimately, it remains unclear which of these differences contributed to the disparate study outcomes in these small phase 2 studies.

Although there was an initial increase in total and mature B cells, this was followed by a progressive decrease until week 16 despite declines in serum tabalumab. Further work is needed to better understand why a clinical response was observed before significant reduction in B cells and why B-cell levels continued to decrease when clinical responses were no longer observed at week 16. Early increases in B cells with subsequent decreases have also been observed with other BAFF-targeted therapies, including briobacept, ${ }^{19}$ atacicept ${ }^{20}$ and belimumab. ${ }^{21}$ In the previous tabalumab trial, ${ }^{14}$ a transient increase in B-cell levels was followed by a sustained decrease until week 24 , although, unlike the present trial, clinical efficacy was maintained until week 24.

The success of other biological RA therapies that target B cells has varied. Reductions in the clinical signs of RA, relative to placebo, have been observed with rituximab ${ }^{322}$ and ocrelizumab ${ }^{23}$ (anti-CD20 antibodies) and belimumab ${ }^{24}$ (a monoclonal antibody that neutralises soluble BAFF). Although statistically significant, improvement observed with belimumab was minimal, ${ }^{24}$ and, in a subgroup analysis of TNF inhibitor-experienced patients, no difference between belimumab and placebo was detected in ACR20 response rate. ${ }^{25}$ Another B-cell-targeted therapy, atacicept (a fusion protein targeting BAFF and APRIL), failed to achieve clinical benefits in two 26 -week trials. ${ }^{26} 27$

A limitation of this trial is that tabalumab was only dosed three times, and it appears that the concentration may have been insufficient to sustain a response for 10 weeks after the last administered dose. This choice was based on data from a previous phase $1 \mathrm{~b}$ study in TNF inhibitor-naïve patients with RA, which showed that maximal clinical efficacy was sustained until at least 18 weeks after the last infusion. This was considered consistent with the observed long-lasting reduction in circulating B-cell levels. Thus, the present trial intended to investigate the effects and duration of efficacy of a comparable treatment regimen in a different population (TNF-IR patients). Another limitation is that it was a small, short-term phase 2 trial limited to TNF-IR patients who were RF or anti-CCP positive.

The primary end point of this study was not met, as no clinical effect was detected at week 16. Clinical efficacy was observed during the peak of drug exposure, but disappeared after tabalumab withdrawal, despite the fact that B cells continued to decrease. Large, placebo-controlled, randomised, phase 3 clinical trials using tabalumab in both MTX-IR and TNF-IR patients from around the world, which are currently underway, should help us to better understand the use of tabalumab in patients with RA.

Acknowledgements The authors would like to thank all of the participating investigators, and also Kelly Guerrettaz of PharmaNet/i3, an inVentiv Health Company (Blue Bell, PA) for writing assistance.

Contributors MCG contributed to the study design and analysis, and reviewed/edited the manuscript; RMF contributed to the study design and analysis, participated as an investigator, and reviewed/edited the manuscript; MG participated as an investigator and reviewed/edited the manuscript; JS, MV and LX contributed to the data analysis and reviewed/edited the manuscript; P-YB, SM and OB contributed to the study design, implementation, and analysis, and reviewed/edited the manuscript.

Funding This study was sponsored by Eli Lilly and Company or Lilly USA, LLC.

Competing interests MCG, RF and MG have received grants and consulting fees from Eli Lilly and Company. JS, MV, LX, P-YB, SM and OB are employees and stockholders of Eli Lilly and Company.
Ethics approval The institutional review boards at each study site.

Patient consent Obtained.

Provenance and peer review Not commissioned; externally peer reviewed.

\section{REFERENCES}

1. American College of Rheumatology Subcommittee on Rheumatoid Arthritis Guidelines. Guidelines for the management of rheumatoid arthritis: 2002 Update. Arthritis Rheum 2002;46:328-46.

2. Singh JA, Christensen R, Wells GA, et al. A network meta-analysis of randomized controlled trials of biolgocis for rheumatoid arthritis: a Cochrane review. CMAJ 2009;181:787-96.

3. Cohen SB, Emery P, Greenwald MW, et al. Rituximab for rheumatoid arthritis refractory to anti-tumor necrosis factor therapy: results of a multicenter, randomized, double-blind, placebo-controlled, phase III trial evaluating primary efficacy and safety at twenty-four weeks. Arthritis Rheum 2006;54:2793-806.

4. Emery P, Keystone E, Tony HP, et al. IL-6 receptor inhibition with tocilizumab improves treatment outcomes in patients with rheumatoid arthritis refractory to anti-tumor necrosis factor biological: results from a 24-week multicentre randomised placebo-controlled trial. Ann Rheum Dis 2008;67:1516-23.

5. Genovese MC, Becker JC, Schiff M, et al. Abatacept for rheumatoid arthritis refractory to tumor necrosis factor $\alpha$ inhibition. N Engl J Med 2005;353:1114-23.

6. Smolen JS, Kay J, Doyle MK, et al. Golimumab in patients with active rheumatoid arthritis after treatment with tumour necrosis factor alpha inhibitors (GO-AFTER study): a multicentre, randomised, double-blind, placebo-controlled, phase III,trial. Lancet 2009:374:210-21.

7. Schneider P, Mackay F, Steiner V, et al. BAFF, a novel ligand of the tumor necrosis factor family, stimulates B cell growth. J Exp Med 1999;189:1747-56.

8. Melchers F. Actions of BAFF in B cell maturation and its effects on the development of autoimmune disease. Ann Rheum Dis 2003;62(Suppl 2):ii25-7.

9. Cancro MP, D'Cruz DP, Khamashta MA. The role of B lymphocyte stimulator (BLyS) in systemic lupus erythematosus. J Clin Invest 2009;119:1066-73.

10. Davidson A. Targeting BAFF in autoimmunity. Curr Opin Immuno/ 2010;22:732-9.

11. Ohata J, Zvaifler NJ, Nishio M, et al. Fibroblast-like synoviocytes of mesenchymal origin express functional $B$ cell-activating factor of the TNF family in response to proinflammatory cytokines. J Immunol 2005;174:864-70.

12. Bosello S, Youinou P, Daridon C, et al. Concentrations of BAFF correlate with autoantibody levels, clinical disease activity, and response to treatment in early rheumatoid arthritis. J Rheumatol 2008;35:1256-64.

13. Kikly K, Manetta J, Smith H, et al. Characterization of LY2127399, a neutralizing antibody for BAFF (abstract). Arthritis Rheum 2009:60(Suppl 10):693.

14. Genovese MC, Mociran E, Biagini M, et al. Phase 2 study of safety and efficacy of a novel anti-BAFF monoclonal antibody, in patients with RA treated with methotrexate (MTX) (abstract). Arthritis Rheum 2009:60(Suppl 10):1923.

15. Arnett FC, Edworthy SM, Bloch DA, et al. The American Rheumatism Association 1987 revised criteria for the classification of rheumatoid arthritis. Arthritis Rheum 1988;31:315-24.

16. Felson DT, Anderson JJ, Boers M, et al. American College of Rheumatology. Preliminary definition of improvement in rheumatoid arthritis. Arthritis Rheum 1995;38:727-35.

17. Van Gestel AM, Prevoo MLL, Van'T Hof MA, et al. Development and validation of the European League Against Rheumatism response criteria for rheumatoid arthritis. Arthritis Rheum 1996;39:34-40.

18. Keystone EC, van der Heijde D, Smolen J, et al. Open-label treatment effect in patients who withdrew from double-blind treatment with certolizumab pegol: substantial improvement in patients receiving active treatment who did not respond in the double-blind phase. Ann Rheum Dis 2009;68:236.

19. Shaw M, Del Giudice J, Trapp R, et al. The safety, pharmacokinetics, and pharmacodynamic effects of repeated doses of BR3-Fc in patients with rheumatoid arthritis. Program and abstracts of the American College of Rheumatology (ACR) 71st Annual Meeting; November 6-11, 2007; Boston, Massachusetts. (Abstract \#1432)

20. Tak PP, Thurlings RM, Rossier C, et al. Atacicept in patients with rheumatoid arthritis: results of a multicenter, phase $\mathrm{lb}$, double-blind, placebo-controlled, dose-escalating, single- and repeated-dose study. Arthritis Rheum 2008;58:61-72.

21. Stohl W, Chatham W, Weisman $\mathbf{M}$, et al. Belimumab, a novel fully human monoclonal antibody to B-lymphocyte stimulator (BLys), selectively modulates B-cell subpopulations and immunoglobulins in a heterogeneous rheumatoid arthritis subject population (abstract). In: Program and Abstracts of the American College of Rheumatology (ACR) 69th Annual Meeting; 2005 Nov 12-17; San Diego, CA: Abstract 1160.

22. Emery P, Fleischmann R, Filipowicz-Sosnowska A, et al. The efficacy and safety of rituximab in patients with active rheumatoid arthritis despite methotrexate treatment. Arthritis Rheum 2006;54:1390-400.

23. Genovese MC, Kaine JL, Lowenstein MB, et al. Ocrelizumab, a humanized anti-CD20 monoclonal antibody, in the treatment of patients with rheumatoid arthritis. Arthritis Rheum 2008;58:2652-1. 
24. McKay J, Chwalinska-Sadowska H, Boling E, et al. Belimumab (BmAb), a fully human monoclonal antibody to B-lymphocyte stimulator (BLyS), combined with standard of care therapy reduces the signs and symptoms of rheumatoid arthritis in a heterogeneous subject population. Arthritis Rheum 2005;52:S710.

25. Genovese MC, Filipowicz-Sosnowska A, Merrill J, et al. Differential responsiveness of belimumab (BmAb) in combination with standard of care therapy in RF+, TNFa inhibitor and MTX partial responder subgroups with moderate-severe rheumatoid arthritis (abstract). Arthritis Rheum 2005;52:S1989.
26. Genovese MC, Kinnman N, de La BG, et al. Atacicept in patients with rheumatoid arthritis and an inadequate response to tumor necrosis factor antagonist therapy: results of a phase II, randomized, placebo-controlled, dose-finding trial. Arthritis Rheum 2011;63:1793-803.

27. van Vollenhoven RF, Kinnman N, Vincent E, et al. Atacicept in patients with rheumatoid arthritis and an inadequate response to methotrexate: results of a phase II, randomized, placebo-controlled trial. Arthritis Rheum 2011;63:1782-92. 